\title{
On strictly convex central configurations of the $2 n$-body problem
}

\author{
E. Barrabés J.M. Cors
}

May 29, 2018

\begin{abstract}
We consider planar central configurations of the Newtonian $2 n$-body problem consisting in two twisted regular $n$-gons of equal masses. We prove the conjecture that for $n \geq 5$ all convex central configurations of two twisted regular $n$-gons are strictly convex.
\end{abstract}

\section{Introduction and Main Result}

Consider the planar Newtonian $2 n$-body problem of two groups of $n$ bodies in the same plane $(x, y)$ at positions $\mathbf{q}_{j i} \in \mathbb{R}^{2}, i=1, \ldots, n, j=1,2$, such that all the bodies in the same group have equal mass, $m_{1}$ and $m_{2}$, respectively. Without lost of generality, we can consider the center of mass at the origin. It is well known (see Saari (2005)) that a central configuration of the planar $2 n$-body problem is a solution $\mathbf{q}=\left(\mathbf{q}_{11}, \mathbf{q}_{12}, \ldots, \mathbf{q}_{2 n}\right) \in \mathbb{R}^{4 n}$ of the equation

$$
\nabla U(\mathbf{q})+w^{2} M \mathbf{q}=0
$$

for some value of $w$, where $U$ is the Newtonian potential

$$
U(\mathbf{q})=\sum_{j=1}^{2} \sum_{i=1}^{n-1} \sum_{l=i+1}^{n} \frac{m_{j}^{2}}{\left\|\mathbf{q}_{j i}-\mathbf{q}_{j l}\right\|}+\sum_{i=1}^{n} \sum_{m=1}^{n} \frac{m_{1} m_{2}}{\left\|\mathbf{q}_{1 i}-\mathbf{q}_{2 m}\right\|},
$$

and $M$ is the diagonal mass matrix with diagonal $m_{1}, \ldots, m_{1}, m_{2}, \ldots, m_{2}$ (each mass repeated $n$ times).

We are interested in central configurations such that the bodies are located at the vertices of two regular $n$-gons. A configuration of $n$ equal masses at the vertices of a regular $n$-gon is also called a ring. Yu and Zhang (2012) show that in any central configuration of two regular $n$-gons of equal masses within each gon, the two rings are nested, i.e., the vertices of the two $n$-gons are aligned, or they are twisted, that is, the vertices of the second $n$-gon are rotated an angle $\pi / n$ with respect the vertices of the first one.

Definition 1. Let $\mathbf{q}_{j i} \in \mathbb{R}^{2}, i=1, \ldots, n, j=1,2$ be a central configuration of two rings. The configuration is convex if the interior of the polygon defined by the sequence of vertex $\mathbf{q}_{11}, \mathbf{q}_{21}, \mathbf{q}_{12}, \mathbf{q}_{22}, \ldots, \mathbf{q}_{1 \mathbf{n}}, \mathbf{q}_{2 \mathbf{n}}$ is a convex set. It is strictly convex if every internal angle is strictly less than $\pi$.

Clearly, from the definition, in a convex central configuration of two rings each vertex $\mathbf{q}_{\mathbf{j i}}$ is on the boundary of the convex hull defined by all the vertices. Moreover, a configuration is convex but not strictly convex if one or more interior angles are equal to $\pi$. 
Since no convex configurations are possible when the two rings are nested, we focuss on central configurations of two twisted rings. Without lost of generality, we can consider that the bodies of one ring have mass $m_{1}=1$ and are located on a circle of radius 1 . The second ring is formed by bodies of mass $m_{2}=m$ and are located at a circle of radius $r$. It is not difficult to see that the configuration is convex if and only if

$$
a_{n}=\cos \left(\frac{\pi}{n}\right) \leq r \leq \frac{1}{\cos \left(\frac{\pi}{n}\right)}=\frac{1}{a_{n}} .
$$

The values of $a_{n}, 1 / a_{n}$ correspond to convex configurations which are not strictly convex. See Figure 1.

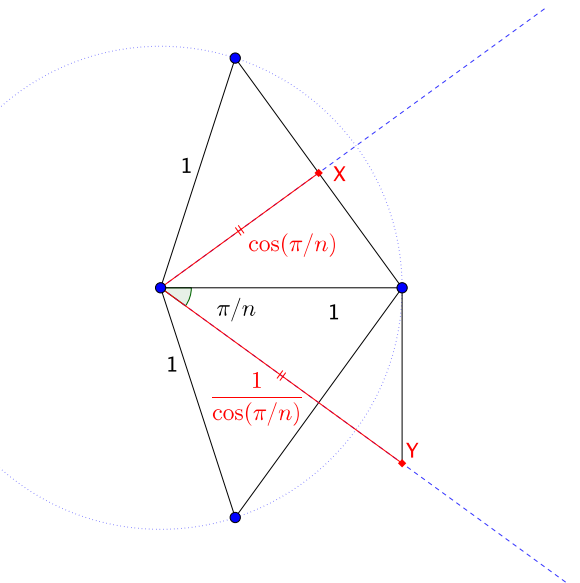

Figure 1: The points $X$ and $Y$ give the limits for the strictly convex configurations of two rings.

In Roberts (1999), Yu and Zhang (2012) and Barrabés and Cors (2016), it is shown that two twisted rings are in central configuration if and only if $m$ and $r$ satisfy the following equation:

$$
(C(r)-r S)+\left(\frac{S}{r^{2}}-r D(r)\right) m=0
$$

where $S$ is the constant

$$
S=\frac{1}{4} \sum_{k=1}^{n-1} \frac{1}{\sin (k \pi / n)}
$$

and $C$ and $D$ are functions of $r$ that depend on mutual distances:

$$
\begin{aligned}
& C(r)=\sum_{k=1}^{n} \frac{r-\cos ((2 k-1) \pi / n)}{\left(1+r^{2}-2 r \cos ((2 k-1) \pi / n)\right)^{3 / 2}}, \\
& D(r)=\sum_{k=1}^{n} \frac{1-r \cos ((2 k-1) \pi / n)}{\left(1+r^{2}-2 r \cos ((2 k-1) \pi / n)\right)^{3 / 2}} .
\end{aligned}
$$

Notice that equation (2) is linear with respect to the mass $m$. Then, solving for $m$ as a function of the radius $r>0$ we have that

$$
m=H(r)=\frac{r^{2}(r S-C(r))}{S-r^{3} D(r)}=r^{2} \frac{F(r)}{G(r)} .
$$

Therefore, not all values of $r$ are admissible, only those for which $H(r)>0$. In Barrabés and Cors (2016), we characterize the admissible values of $r$ and the number of central configurations for each value of $n$. 
In Figure 2 we show two central configurations of two twisted rings for $n=4$ and $n=5$. For $n=4$, a solution of equation (6) is given by $r=\sqrt{2}$ and $m=16.05679942 \ldots$ that corresponds a convex central configuration of the 8-body problem which is not strictly convex. For $n=5$, a solution of equation (6) is given by $r=1.1$ and $m=2.365332699 \ldots$, which is a strictly convex central configuration of the 10-body problem. In the general $N$-body problem, it is known that when $N=4$ any convex central configuration is strictly convex. In the 5-body problem an example of a convex central configuration which is not strictly convex is given by Chen and Hsiao (2012).
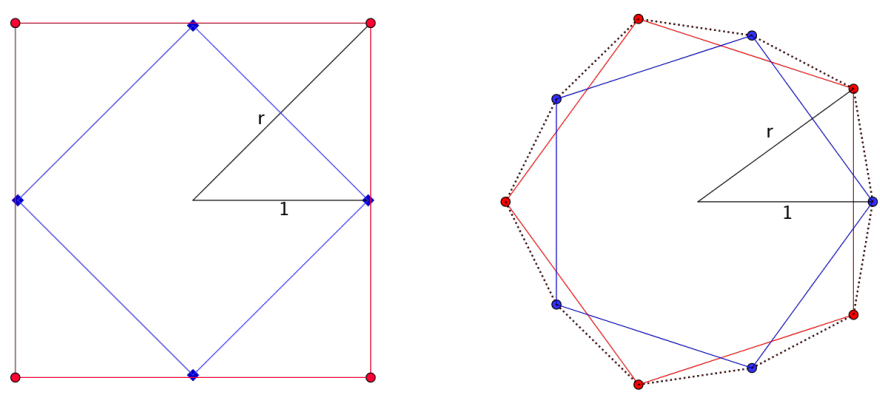

Figure 2: Left, a convex central configuration of the 8-body problem which is not strictly convex formed by two twisted rings with $r=\sqrt{2}$. Right, a strictly convex central configuration of the 10-body problem formed by two twisted rings with with $r=1.1$

In Fernandes et al. (2017) the authors prove that any convex central configuration of two twisted rings when $n=3,5,6$ is strictly convex. For $n=4$ they show that there exists a convex central configuration which is not strictly convex (represented in Figure 2, left). They also conjecture that $n=4$ is the only value of $n$ for which there exist non-strictly convex central configurations of two twisted rings. Our aim is to give proof of that conjecture.

Theorem 1. Let us consider $2 n$ bodies in a central configuration of two twisted rings. Then, for $n \geq 5$ all convex central configurations are strictly convex.

All the numerical computations carried out throughout the paper use integer arithmetics. For instance, in the computation of the Sturm sequence of a univariate polynomial with integer coefficients to count the number of real roots in a given interval. Since no floating-point arithmetic have been used nowhere, all the results are analytic.

\section{Proof of Theorem 1}

First, we prove that the set of strictly convex central configurations of two twisted rings is not empty. In fact, $r=1$ and $m=1$ is a solution of (6) that correspond to the regular $2 n$-gon with equal masses, which is obviously a strictly convex central configuration.

Second, recall from (1) that the values $r=a_{n}, 1 / a_{n}$ correspond to convex configurations which are not strictly convex. Therefore, to proof Theorem 1 it is enough to prove that both $H\left(a_{n}\right)<0$ and $H\left(1 / a_{n}\right)<0$, where $m=H(r)$ is the function defined in (6).

Next result comes straightforward from the definitions of the functions $F$ and $G$ in (6).

Proposition 1. Let $F(r)$ and $G(r)$ be the functions defined in (6) for $r \in(0, \infty)$. Then, $F(r)$ and $G(r)$ are analytic functions and $F(r)=r G(1 / r)$. 
From Proposition 1, for all positive values of $r, H(1 / r)=1 / H(r)$. Thus, the proof of Theorem 1 reduces to see that $H\left(a_{n}\right)<0$. This is equivalently to see that $F\left(a_{n}\right) \cdot G\left(a_{n}\right)<0$, which follows from the next two Propositions.

Proposition 2. Let $F(r)$ be defined in (6) for $r \in(0, \infty)$ and $a_{n}=\cos \left(\frac{\pi}{n}\right)$. Then, $F\left(a_{n}\right)>0$ for $n \geq 5$.

Proposition 3. Let $G(r)$ be defined in (6) for $r \in(0, \infty)$ and $a_{n}=\cos \left(\frac{\pi}{n}\right)$. Then, $G\left(a_{n}\right)<0$ for $n \geq 5$.

Next subsections are devoted to prove Propositions 2 and 3.

\subsection{Proof of Proposition 2}

From equation (6), $F(r)=r S-C(r)$, where $S$ and $C(r)$ are given in (3) and (4). First, notice that due to the symmetry of the angles involved in the sums we can write

$$
S= \begin{cases}\frac{1}{2} \sum_{k=1}^{(n / 2)-1} \frac{1}{\sin (k \pi / n)}+\frac{1}{4} & n \text { even }, \\ \frac{1}{2} \sum_{k=1}^{(n-1) / 2} \frac{1}{\sin (k \pi / n)} & n \text { odd }\end{cases}
$$

and

$$
C(r)= \begin{cases}2 \sum_{k=1}^{n / 2} \frac{r-\cos ((2 k-1) \pi / n)}{\left(1+r^{2}-2 r \cos ((2 k-1) \pi / n)\right)^{3 / 2}} & n \text { even }, \\ 2 \sum_{k=1}^{(n-1) / 2} \frac{r-\cos ((2 k-1) \pi / n)}{\left(1+r^{2}-2 r \cos ((2 k-1) \pi / n)\right)^{3 / 2}}+\frac{1}{(1+r)^{2}} & n \text { odd } .\end{cases}
$$

Let [.] be the floor function. For convenience, we split $S$ in the following way: $S=S_{1}+S_{2}$, where $S_{1}$ keeps the first $\left[\frac{n-1}{2}\right]-2$ terms and $S_{2}$ the remaining ones. Explicitly,

$$
S_{1}=\left\{\begin{array}{cl}
0 & n=5,6, \\
\frac{1}{2} \sum_{k=1}^{\left[\frac{n-1}{2}\right]-2} \frac{1}{\sin (k \pi / n)} & n \geq 7
\end{array}\right.
$$

and

$$
S_{2}=\left\{\begin{array}{cl}
\frac{1}{2}\left(\frac{1}{\sin \left(\left(\frac{n}{2}-2\right) \frac{\pi}{n}\right)}+\frac{1}{\sin \left(\left(\frac{n}{2}-1\right) \frac{\pi}{n}\right)}\right)+\frac{1}{4} & 6 \leq n \text { even } \\
\frac{1}{2}\left(\frac{1}{\sin \left(\left(\frac{n-3}{2}\right) \frac{\pi}{n}\right)}+\frac{1}{\sin \left(\left(\frac{n-1}{2}\right) \frac{\pi}{n}\right)}\right) & 5 \leq n \text { odd }
\end{array}\right.
$$

We also split the function $C(r)=C_{1}(r)+C_{2}(r)$, such that $C_{1}$ keeps the first $\left[\frac{n-1}{2}\right]-1$ terms and $C_{2}$ the remaining ones:

$$
C_{1}(r)=\left\{\begin{array}{cl}
0 & n=5,6, \\
2 \sum_{k=1}^{\left[\frac{n-1}{2}\right]-1} \frac{r-\cos ((2 k-1) \pi / n)}{\left(1+r^{2}-2 r \cos ((2 k-1) \pi / n)\right)^{3 / 2}} & n \geq 7,
\end{array}\right.
$$


and

$$
C_{2}(r)=\left\{\begin{array}{cl}
\frac{2(r-\cos ((n-3) \pi / n))}{\left(1+r^{2}-2 r \cos ((n-3) \pi / n)\right)^{3 / 2}}+\frac{2(r-\cos ((n-1) \pi / n))}{\left(1+r^{2}-2 r \cos ((n-1) \pi / n)\right)^{3 / 2}} & 6 \leq n \text { even, } \\
\frac{2(r-\cos ((n-2) \pi / n))}{\left(1+r^{2}-2 r \cos ((n-2) \pi / n)\right)^{3 / 2}}+\frac{1}{(1+r)^{2}} & 5 \leq n \text { odd. }
\end{array}\right.
$$

Therefore, $F=F_{1}+F_{2}$ where $F_{i}(r)=r S_{i}-C_{i}(r), i=1,2$. Next, we will show that $F_{1}\left(a_{n}\right) \geq 0$ and $F_{2}\left(a_{n}\right)>0$ for $n \geq 5$, which concludes the proof of Proposition 2.

1. First we consider the function $F_{1}(r)$ for $n \geq 7\left(F_{1}=0\right.$ for $\left.n=5,6\right)$. Recall that $a_{n}=$ $\cos (\pi / n)$. Notice that the first sumand in the expression of $C_{1}\left(a_{n}\right)$ vanish. Therefore

$$
F_{1}\left(a_{n}\right)=\sum_{k=1}^{\left[\frac{n-1}{2}\right]-2}\left(\frac{a_{n}}{2 \sin (k \pi / n)}-\frac{2\left(a_{n}-\cos ((2 k+1) \pi / n)\right)}{\left(1+a_{n}^{2}-2 a_{n} \cos ((2 k+1) \pi / n)\right)^{3 / 2}}\right) .
$$

Expanding $\cos ((2 k+1) \pi / n)$ in terms of $x_{n k}=\cos (k \pi / n)$ we get

$$
F_{1}\left(a_{n}\right)=\sum_{k=1}^{\left[\frac{n-1}{2}\right]-2} \frac{1}{\sin (k \pi / n)} \bar{T}\left(a_{n}, x_{n k}\right),
$$

where

$$
\bar{T}(a, x)=\frac{a}{2}-4 y^{2} \frac{a y+b x}{\left(b^{2} y^{2}+(b x+2 a y)^{2}\right)^{3 / 2}},
$$

and $b=\sqrt{1-a^{2}}, y=\sqrt{1-x^{2}}$. For any fixed value $n$, we have that

$$
\cos \left(\left(\left[\frac{n-1}{2}\right]-2\right) \frac{\pi}{n}\right) \leq x_{n k} \leq \cos \left(\frac{\pi}{n}\right), \quad k=1, \ldots,\left[\frac{n-1}{2}\right]-2 .
$$

Thus, we consider the function $\bar{T}\left(a_{n}, x\right)$ defined in $x \in\left[x_{\min }\left(a_{n}\right), a_{n}\right]$, where

$$
x_{\text {min }}\left(a_{n}\right)= \begin{cases}\sqrt{1-a_{n}^{2}}\left(4 a_{n}^{2}-1\right) & \text { for } n \text { even } \\ \sqrt{\frac{1-a_{n}}{2}}\left(4 a_{n}^{2}+2 a_{n}-1\right) & \text { for } n \text { odd }\end{cases}
$$

Clearly, $F_{1}\left(a_{n}\right)>0$ if $\bar{T}\left(a_{n}, x_{n k}\right)>0$ for all $k=1, \ldots,\left[\frac{n-1}{2}\right]-2$ and $n \geq 7$. To show that we use the following Lemma. Its proof can be found in the Appendix.

Lemma 1. For any fixed value $a \in[\cos (\pi / 7), 1)$, let $b=\sqrt{1-a^{2}}$ and

$$
T(a, u)=\frac{a}{2}-\frac{4}{b^{3}} \frac{b u-a}{\left(1+u^{2}\right)^{3 / 2}} .
$$

Then, $T(a, u)>0$ for $u \in\left[\frac{4 a^{3}-2 a^{2}+a-1}{b\left(4 a^{2}-2 a-1\right)}, \frac{3 a}{b}\right]$.

Introducing the new variable $u=x / y+2 a / b$ in the expression (8), we get that $\bar{T}(a, x)=$ $T(a, u)$ where $T$ is the function defined in Lemma 1. Using that $u$ is an increasing function of $x$, the interval $x \in\left[x_{\min }\left(a_{n}\right), a_{n}\right]$ transforms into $u \in\left[u_{\min }\left(a_{n}\right), 3 a_{n} / b_{n}\right]$ where

$$
u_{\text {min }}(a)= \begin{cases}u_{m}^{e}(a)=\frac{4 a^{4}-a^{2}-1}{a b\left(4 a^{2}-3\right)} & \text { for } n \text { even, } \\ u_{m}^{o}(a)=\frac{4 a^{3}-2 a^{2}+a-1}{b\left(4 a^{2}-2 a-1\right)} & \text { for } n \text { odd. }\end{cases}
$$


It is important to notice that to derive the expressions of $u_{\min }(a)$ we have used that $4 a^{2}-3>0$ ( $n$ even) and $4 a^{2}-2 a-1>0$ ( $n$ odd), which are true for $a>\cos (\pi / 8)(n$ even) or $a>\cos (\pi / 7)$ $(n$ odd $)$.

It is a straightforward computation to verify that $u_{m}^{o}(a)<u_{m}^{e}(a)$ when $a<1$ (see Figure 3). Thus, using the result of Lemma 1 , we finish the proof that $F_{1}\left(a_{n}\right)>0$.

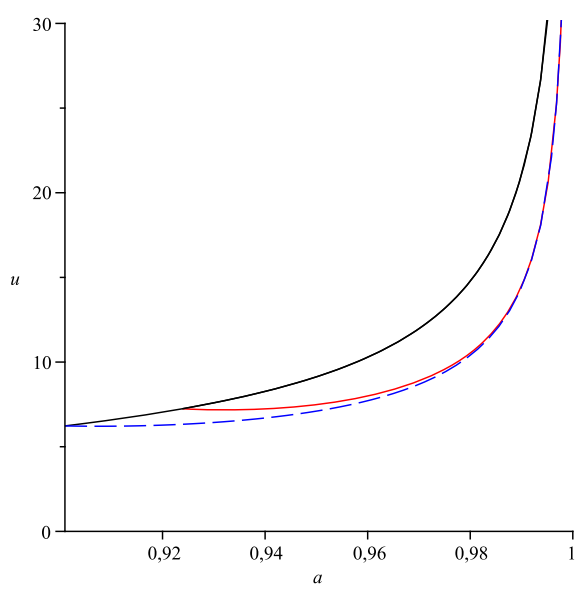

Figure 3: Domain of $T(a, u)$ given in Lemma 1: $u_{m}^{e}(a)$, red solid curve; $u_{m}^{o}(a)$, blue dashed curve, are given by (9) and $u=3 a / b$, black solid curve.

2. Second, we want to show that $F_{2}\left(a_{n}\right)>0$ for $n \geq 6$ even. As before, recall that $a_{n}=$ $\cos (\pi / n)$. Therefore, $F_{2}\left(a_{n}\right)=q_{1}\left(a_{n}\right)-q_{2}\left(a_{n}\right)$, where

$$
\begin{aligned}
q_{1}(a) & =\frac{1}{2}+\frac{a}{4}+\frac{a}{2\left(2 a^{2}-1\right)}, \\
q_{2}(a) & =\frac{4 a\left(2 a^{2}-1\right)}{\left(1-5 a^{2}+8 a^{4}\right)^{3 / 2}}+\frac{4 a}{\left(1+3 a^{2}\right)^{3 / 2}},
\end{aligned}
$$

are continuous decreasing functions in $[\cos (\pi / 8), 1]$. Then,

$$
F_{2}\left(a_{n}\right) \geq q_{1}(1)-q_{2}(\cos (\pi / 8))>0, \quad \text { for } n \geq 8 .
$$

The case $n=6$ can be checked directly.

3. Finally, we want to show that $F_{2}\left(a_{n}\right)>0$ for $n \geq 7$ odd. In this case,

$$
F_{2}\left(a_{n}\right)=\frac{a_{n}}{2 \cos \left(\frac{\pi}{2 n}\right)}\left(\frac{1}{2 a_{n}-1}+1\right)-\frac{2\left(2 a_{n}^{2}+a_{n}-1\right)}{\left(1-2 a_{n}+a_{n}^{2}+4 a_{n}^{3}\right)^{3 / 2}}-\frac{1}{\left(1+a_{n}\right)^{2}} .
$$

It is more suitable to write the above expression in terms of the half angle $p_{n}=\cos \left(\frac{\pi}{2 n}\right)$ through the identity $a_{n}=2 p_{n}^{2}-1$. Thus, $F_{2}\left(a_{n}\right)=q_{1}\left(p_{n}\right)-q_{2}\left(p_{n}\right)$ where

$$
\begin{aligned}
& q_{1}(p)=\frac{\left(2 p^{2}-1\right)^{2}}{p\left(4 p^{2}-3\right)}-\frac{1}{4 p^{4}} \\
& q_{2}(p)=\frac{4 p^{2}\left(4 p^{2}-3\right)}{\left(40 p^{6}-60 p^{4}+26 p^{2}-2\right)^{3 / 2}},
\end{aligned}
$$

for $p \in[\cos (\pi / 14), 1]$. Both functions are continuous and decreasing in that interval, so

$$
F_{2}\left(a_{n}\right) \geq q_{1}(1)-q_{2}(\cos (\pi / 14))>0, \quad \text { for } n \geq 7 .
$$




\subsection{Proof of Proposition 3}

From equation (6), $G(r)=S-r^{3} D(r)$, where $S$ and $D(r)$ are given in (3) and (5). We will follow the same ideas than in the previous Section in order to prove that $G\left(a_{n}\right)<0$.

First, we use the symmetry of the angles to write the constant $S$ as in (7) and

$$
D(r)= \begin{cases}2 \sum_{k=1}^{n / 2} \frac{1-r \cos ((2 k-1) \pi / n)}{\left(1+r^{2}-2 r \cos ((2 k-1) \pi / n)\right)^{3 / 2}} & n \text { even }, \\ 2 \sum_{k=1}^{(n-1) / 2} \frac{1-r \cos ((2 k-1) \pi / n)}{\left(1+r^{2}-2 r \cos ((2 k-1) \pi / n)\right)^{3 / 2}}+\frac{1}{(1+r)^{2}} & n \text { odd. }\end{cases}
$$

Next, we split $S=S_{1}+S_{2}$, where $S_{1}$ keeps the first $\left[\frac{n}{2}\right]-2$ terms and $S_{2}$ the remaining ones. Explicitly, for $n \geq 5$

$$
S_{1}= \begin{cases}0 & n=5 \\ \frac{1}{2} \sum_{k=1}^{\left[\frac{n}{2}\right]-2} \frac{1}{\sin (k \pi / n)} & n \geq 6\end{cases}
$$

and

$$
S_{2}=\left\{\begin{array}{cl}
\frac{1}{2} \frac{1}{\sin \left(\left(\frac{n}{2}-1\right) \frac{\pi}{n}\right)}+\frac{1}{4} & 6 \leq n \text { even }, \\
\frac{1}{2}\left(\frac{1}{\sin \left(\left(\frac{n-3}{2}\right) \frac{\pi}{n}\right)}+\frac{1}{\sin \left(\left(\frac{n-1}{2}\right) \frac{\pi}{n}\right)}\right) & 5 \leq n \text { odd }
\end{array}\right.
$$

We also split the function $D(r)=D_{1}(r)+D_{2}(r)$, such that $D_{1}$ keeps the first $\left[\frac{n}{2}\right]-2$ terms and $D_{2}$ the remaining ones:

$$
D_{1}(r)=\left\{\begin{array}{cl}
0 & n=5, \\
2 \sum_{k=1}^{\left[\frac{n}{2}\right]-2} \frac{1-r \cos ((2 k-1) \pi / n)}{\left(1+r^{2}-2 r \cos ((2 k-1) \pi / n)\right)^{3 / 2}} & n \geq 6,
\end{array}\right.
$$

and

$$
D_{2}(r)=\left\{\begin{array}{cc}
\frac{2(1-r \cos ((n-3) \pi / n))}{\left(1+r^{2}-2 r \cos ((n-3) \pi / n)\right)^{3 / 2}}+\frac{2(1-r \cos ((n-1) \pi / n))}{\left(1+r^{2}-2 r \cos ((n-1) \pi / n)\right)^{3 / 2}} & 6 \leq n \text { even, } \\
\frac{2(1-r \cos ((n-4) \pi / n))}{\left(1+r^{2}-2 r \cos ((n-4) \pi / n)\right)^{3 / 2}}+\frac{2(1-r \cos ((n-2) \pi / n))}{\left(1+r^{2}-2 r \cos ((n-2) \pi / n)\right)^{3 / 2}}+\frac{1}{(1+r)^{2}} & 5 \leq n \text { odd } .
\end{array}\right.
$$

Therefore, $G=G_{1}+G_{2}$ where $G_{i}(r)=S_{i}-r^{3} D_{i}(r), i=1,2$. Next, we will show that $G_{1}\left(a_{n}\right) \leq 0$ and $G_{2}\left(a_{n}\right)<0$ for $n \geq 5$. Recall that $a_{n}=\cos (\pi / n)$.

1. First we consider the function $G_{1}(r)$ for $n \geq 6\left(G_{1}=0\right.$ for $\left.n=5\right)$, which can be written as

$$
G_{1}\left(a_{n}\right)=\sum_{k=1}^{\left[\frac{n}{2}\right]-2}\left(\frac{1}{2 \sin (k \pi / n)}-\frac{2 a_{n}^{3}\left(1-a_{n} \cos ((2 k-1) \pi / n)\right)}{\left(1+a_{n}^{2}-2 a_{n} \cos ((2 k-1) \pi / n)\right)^{3 / 2}}\right) .
$$

Expanding $\cos ((2 k-1) \pi / n)$ in terms of $x_{n k}=\cos (k \pi / n)$ we get

$$
G_{1}\left(a_{n}\right)=\sum_{k=1}^{\left[\frac{n}{2}\right]-2} \frac{1}{\sin (k \pi / n)} \bar{T}\left(a_{n}, x_{n k}\right)
$$


where

$$
\bar{T}(a, x)=\frac{1}{2}-2 a^{3} y \frac{y^{2}+(a y-b x)^{2}}{\left(b^{2} y^{2}+(2 a y-b x)^{2}\right)^{3 / 2}},
$$

and $b=\sqrt{1-a^{2}}, y=\sqrt{1-x^{2}}$. For any fixed value $n$, we have that

$$
\cos \left(\left(\left[\frac{n}{2}\right]-2\right) \frac{\pi}{n}\right) \leq x_{n k} \leq \cos \left(\frac{\pi}{n}\right), \quad k=1, \ldots,\left[\frac{n-1}{2}\right]-2 .
$$

Therefore, it is enough to see that $\bar{T}\left(a_{n}, x\right)<0$ for $x \in\left[x_{\min }\left(a_{n}\right), a_{n}\right]$ where

$$
x_{\min }\left(a_{n}\right)= \begin{cases}2 a_{n} b_{n} & \text { for } n \text { even, } \\ \sqrt{\frac{1-a_{n}}{2}}\left(4 a_{n}^{2}+2 a_{n}-1\right) & \text { for } n \text { odd. }\end{cases}
$$

In order to do that we use the following Lemma. Its proof can be found in the Appendix.

Lemma 2. For any fixed value $a \in[\cos (\pi / 6), 1)$, let $b=\sqrt{1-a^{2}}$ and

$$
T(a, u)=\frac{1}{2}-2 \frac{a^{2}}{b^{3}} \frac{1+(b u-a)^{2}}{\left(1+u^{2}\right)^{3 / 2}} .
$$

Then, $T(a, u)<0$ for $u \in\left[\frac{a}{b}, \frac{2 a\left(3 a^{2}-2\right)}{b\left(2 a^{2}-1\right)}\right]$.

Introducing the new variable $u=-x / y+2 a / b$ in the expression (10), we get that $\bar{T}(a, x)=$ $T(a, u)$ where $T$ is the function defined in Lemma 2. Using that $u$ is a decreasing function of $x$, the interval $x \in\left[x_{\min }\left(a_{n}\right), a_{n}\right]$ transforms into $u \in\left[a_{n} / b_{n}, u_{\max }\left(a_{n}\right)\right]$ where

$$
u_{\max }(a)= \begin{cases}u_{m}^{e}(a)=\frac{2 a\left(3 a^{2}-2\right)}{b\left(2 a^{2}-1\right)} & \text { for } n \text { even } \\ u_{m}^{o}(a)=\frac{12 a^{3}-6 a^{2}-5 a+1}{b\left(4 a^{2}-2 a-1\right)} & \text { for } n \text { odd }\end{cases}
$$

It is important to notice that to derive the expressions of $u_{\max }(a)$ we have used that $2 a^{2}-1>$ 0 ( $n$ even) and $4 a^{2}-2 a-1>0(n$ odd $)$, which are true for $a>\cos (\pi / 6)$ ( $n$ even) or $a>\cos (\pi / 7)(n$ odd $)$.

It is a straightforward computation to verify that $u_{m}^{o}(a)<u_{m}^{e}(a)$ when $a<1$ (see Figure 4). So, using the result of Lemma 2, we finish the proof that $G_{1}\left(a_{n}\right)>0$ for $n \geq 6$.

2. Second, we want to show that $G_{2}\left(a_{n}\right)<0$ for $n \geq 6$ even. Using that $a_{n}=\cos (\pi / n)$, $G_{2}\left(a_{n}\right)=q_{1}\left(a_{n}\right)-q_{2}\left(a_{n}\right)$, where

$$
\begin{aligned}
q_{1}(a) & =\frac{1}{4}+\frac{1}{2 a}-\frac{2 a^{3}\left(1+a^{2}\right)}{\left(1+3 a^{2}\right)^{3 / 2}} \\
q_{2}(a) & =\frac{2 a^{3}\left(4 a^{4}-3 a^{2}+1\right)}{\left(8 a^{4}-5 a^{2}+1\right)^{3 / 2}}
\end{aligned}
$$

are continuous decreasing functions in $[\cos (\pi / 6), 1]$. Then,

$$
G_{2}\left(a_{n}\right) \leq q_{1}(\cos (\pi / 6))-q_{2}(1)=\frac{\sqrt{3}}{3}+\frac{1}{4}-\frac{21 \sqrt{39}}{338}-\frac{1}{2}<0 .
$$

3. Finally, we want to show that $G_{2}\left(a_{n}\right)<0$ for $n \geq 5$ odd. In this case,

$$
\begin{aligned}
G_{2}\left(a_{n}\right) & =\frac{a_{n}}{\cos \left(\frac{\pi}{2 n}\right)\left(2 a_{n}-1\right)}-\frac{a_{n}^{3}}{\left(1+a_{n}\right)^{2}} \\
& -2 a_{n}^{3}\left(\frac{2 a_{n}^{3}-a_{n}+1}{\left(4 a_{n}^{3}+a_{n}^{2}-2 a_{n}+1\right)^{3 / 2}}-\frac{8 a_{n}^{5}-8 a_{n}^{3}+a_{n}+1}{\left(16 a_{n}^{5}-16 a_{n}^{3}+a_{n}^{2}+2 a_{n}+1\right)^{3 / 2}}\right) .
\end{aligned}
$$




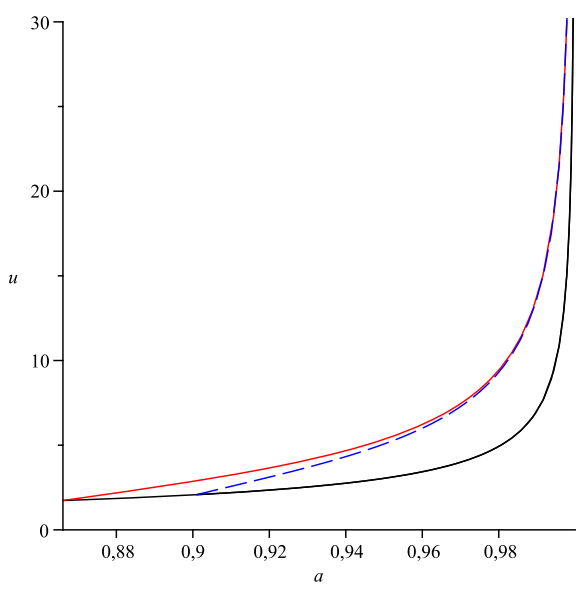

Figure 4: Domain of $T(a, u)$ given in Lemma 2: $u_{m}^{e}(a)$, red solid curve; $u_{m}^{o}(a)$, blue dashed curve, are given by (11). $u=a / b$, black solid curve.

It is more suitable to write the above expression in terms of the half angle $p_{n}=\cos \left(\frac{\pi}{2 n}\right)$ through the identity $a_{n}=2 p_{n}^{2}-1$. Then,

$$
G_{2}\left(a_{n}\right)=\frac{\left(2 p_{n}^{2}-1\right)}{2 p_{n}}\left(q_{1}\left(p_{n}\right)-q_{2}\left(p_{n}\right)\right)
$$

where

$$
\begin{aligned}
& q_{1}(p)=-\left(2 p^{2}-1\right)^{2}\left(\frac{1}{2 p^{3}}+\frac{8 p^{4}-12 p^{2}+5}{\left(8 p^{4}-11 p^{2}+4\right)^{3 / 2}}\right), \\
& q_{2}(p)=-\frac{2}{4 p^{2}-3}+\left(2 p^{2}-1\right)^{2} \frac{128 p^{8}-320 p^{6}+288 p^{4}-112 p^{2}+17}{\left(128 p^{8}-320 p^{6}+288 p^{4}-111 p^{2}+16\right)^{3 / 2}} .
\end{aligned}
$$

It is not difficult to see that $q_{1}$ decreases and that $q_{2}(p)>-11 / 10$ for $p \in\left[\cos \left(\frac{\pi}{10}\right), 1\right]$. Therefore,

$$
\begin{aligned}
q_{1}\left(p_{n}\right)-q_{2}\left(p_{n}\right) & <q_{1}\left(\cos \left(\frac{\pi}{10}\right)\right)+\frac{11}{10} \\
& =-\frac{\sqrt{10+2 \sqrt{5}}}{10}-\frac{\sqrt{14-2 \sqrt{5}}}{484}(85+31 \sqrt{5})+\frac{11}{10}<0 .
\end{aligned}
$$

This concludes the proof.

\section{Appendix}

\subsection{Proof of Lemma 1}

We want to prove that, for any fixed value $a \in[\cos (\pi / 7), 1)$, the function

$$
T(a, u)=\frac{a}{2}-\frac{4}{b^{3}} \frac{b u-a}{\left(1+u^{2}\right)^{3 / 2}},
$$

is positive in the closed interval $u \in \mathcal{K}=\left[u_{m}(a), \frac{3 a}{b}\right]$, where $b=\sqrt{1-a^{2}}$ and

$$
u_{m}(a)=\frac{4 a^{3}-2 a^{2}+a-1}{b\left(4 a^{2}-2 a-1\right)} .
$$


The function $T$ is clearly continuous and differentiable in the domain, so $T$ must attain an absolute minimum on $\mathcal{K}$. On one hand, if we look for critical points in the interior of $\mathcal{K}$,

$$
\frac{\partial T}{\partial u}=\frac{-4}{b^{3}} \frac{b-2 b u^{2}+3 a u}{\left(1+u^{2}\right)^{5 / 2}}=0
$$

occurs at $u_{c}=\left(3 a+\sqrt{a^{2}+8}\right) /(4 b)$. It is not difficult to see that $u_{c}<u_{m}$ for $a \in[\cos (\pi / 7), 1)$, and $\partial T / \partial u(a, u)>0$ for $u \in\left[u_{m}, 3 a / b\right]$. Therefore, the minima of $T$ occurs at $u=u_{m}(a)$.

To conclude the proof it is enough to see that $T\left(a, u_{m}(a)\right)>0$. Simplifying, we want to see that

$$
T\left(a, u_{m}(a)\right)=\frac{a}{2}-\sqrt{2} \frac{(2 a-1)\left(4 a^{2}-2 a-1\right)^{2}}{\left(16 a^{4}-16 a^{3}+a-1\right)^{3 / 2}}>0,
$$

which is equivalent to prove that

$$
a\left(16 a^{4}-16 a^{3}+a-1\right)^{3 / 2}>2 \sqrt{2}(2 a-1)\left(4 a^{2}-2 a-1\right)^{2} .
$$

Squaring both sides, the expression factors into

$$
(a-1) p(x)>0,
$$

where $p(x)$ is a polynomial of degree 13 with integer coefficients, which using Sturm's Theorem contains no real zeros in the interval $[9 / 10,1]$.

\subsection{Proof of Lemma 2}

We want to prove that, for any fixed value of $a \in[\cos (\pi / 6), 1)$, the function

$$
T(a, u)=\frac{1}{2}-2 \frac{a^{2}}{b^{3}} \frac{1+(b u-a)^{2}}{\left(1+u^{2}\right)^{3 / 2}}
$$

is negative for any $u \in \mathcal{K}=\left[\frac{a}{b}, u_{m}(a)\right]$, where $b=\sqrt{1-a^{2}}$ and

$$
u_{m}(a)=\frac{2 a\left(3 a^{2}-2\right)}{b\left(2 a^{2}-1\right)} .
$$

The function is clearly continuous and differentiable in $\mathcal{K}$. On one hand, the function $T(a, u)$ is strictly increasing with respect the variable $u$ because

$$
\begin{aligned}
\frac{\partial T}{\partial u} & =\frac{2 a^{3}}{b^{3}} \frac{b^{2} u^{3}-4 a b u^{2}+\left(5 a^{2}+1\right) u+2 a b}{\left(1+u^{2}\right)^{5 / 2}} \\
& \geq \frac{2 a^{3}}{b^{3}} \frac{u\left(b^{2} u^{2}-4 a b u+5 a^{2}+1\right.}{\left(1+u^{2}\right)^{5 / 2}}>0 .
\end{aligned}
$$

Therefore, $T(a, u) \leq T\left(a, u_{m}(a)\right)$ and

$$
p(a)=T\left(a, u_{m}\right)=\frac{1}{2}-2 a^{3} \frac{\left(2 a^{2}-1\right)\left(16 a^{6}-20 a^{4}+5 a^{2}+1\right)}{\left(32 a^{6}-40 a^{4}+11 a^{2}+1\right)^{3 / 2}} .
$$

Finally, it is not difficult to see that $p^{\prime}(a)$ has no roots in $a \in[\cos (\pi / 6), 1]$ (for example, using Sturm's theory) and $p^{\prime}(a)>0$. Therefore, $T\left(a, u_{m}(a)\right)<p(1)=0$, which concludes the proof.

Acknowledgments: E. Barrabés has been supported by grants MTM2016-80117-P (MINECO/FEDER, UE), and Catalan (AGAUR) grant 2017 SGR 1374. J. M. Cors was partially supported by grants MTM2016-77278-P (FEDER) and AGAUR grant 2017 SGR 1617. 


\section{References}

E Barrabés and J.M. Cors. On central configurations of twisted crowns. arXiv:1612.07135 190.

Chen KC, Hsiao JS (2012) Convex central configurations of the $n$-body problem which are not strictly convex. Journal of Dynamics and Differential Equations 24:119-128

A.C. Fernandes, B.A. Garcia, and L.F. Mello. Convex but not strictly convex central configurations. Journal of Dynamics and Differential Equations, 2017.

G.E. Roberts. Existence and Stability of Relative Equilibria in the N-Body Problem. PhD thesis, Boston University, 1999.

Donald G. Saari. Collisions, rings, and other Newtonian N-body problems, volume 104 of CBMS Regional Conference Series in Mathematics. Published for the Conference Board of the Mathematical Sciences, Washington, DC; by the American Mathematical Society, Providence, RI, 2005.

$\mathrm{X}$. Yu and S. Zhang. Twisted angles for central configurations formed by two twisted regular polygons. J. Differential Equations, 253:2106-2122, 2012. 